Bio - grafía. Escritos sobre la Biología y su Enseñanza. ISSN 2027-1034

Edición Extraordinaria. p.p. 302 - 310

Memorias del IX Encuentro Nacional de Experiencias en Enseñanza de la Biología y la Educación Ambiental. IV Congreso Nacional de Investigación en Enseñanza de la Biología.

\title{
APORTES EN LA COMPRENSIÓN ACERCA DE LA CONSTRUCCIÓN DEL CONOCIMIENTO PROFESIONAL DEL PROFESOR DE BIOLOGÍA.
}

\author{
CONTRIBUTIONS IN THE UNDERST ANDING ABOUT THE CONSTRUCTION OF THE \\ PROFESSIONAL KNOWLEDGE OF THE BIOLOGY TEACHER.
}

\author{
Guillermo Fonseca Amaya ${ }^{1}$
}

\section{RESUMEN}

El Conocimiento Profesional del Profesor (CPP) de ciencias, se ha constituido en una línea de investigación de la didáctica, en razón de la necesidad de comprender como los profesores en formación y en ejercicio construyen un conocimiento singular de la profesión docente. En este sentido, se presentan los resultados de un proceso de Investigación - Acción, en donde es posible caracterizar los conocimientos que se integran para construir el Conocimiento Profesional del Profesor de Biología. Así, el CPPB se considera como producto de la integración/transformación entre conocimiento experiencial (historia de vida, contexto, experiencia) y el conocimiento académico (Conocimiento biológico y Conocimiento Didáctico de las ciencias). Este conocimiento se deriva de la reflexión en y sobre la práctica (en relación con la enseñanza y el aprendizaje de los conceptos estructurantes de la biología) que le permiten al estudiante construir explicaciones acerca de los fenómenos naturales de manera contextual y desde allí, promover el cuidado de sí mismo y de las otras especies, y aportar en la comprensión y solución de los problemas socio-ambientales desde una perspectiva compleja.

PALABRAS CLAVE: Conocimiento Profesional del Profesor, Integración de conocimientos, Formación de profesores, Investigación Acción, Estudio de Caso

\section{ABSTRACT}

The Professional Knowledge of the Professor (CPP) of sciences, has been constituted in a line of investigation of the didactics, due to the necessity to understand how the teachers in formation and in exercise construct a unique knowledge of the teaching profession. In this sense, the results of an Action Research process are presented, where it is possible to characterize the knowledge that is integrated to build the Professional Knowledge of the Biology Teacher. Thus, the CPPB is considered as a product of the integration / transformation between experiential knowledge (life history, context, experience) and academic knowledge (Biological

${ }^{1}$ Proyecto Curricular Licenciatura en Biología Universidad Distrital Francisco José de Caldas guifon20@yahoo.com 
Bio - grafía. Escritos sobre la Biología y su Enseñanza. ISSN 2027-1034

Edición Extraordinaria. p.p. 302 - 310

Memorias del IX Encuentro Nacional de Experiencias en Enseñanza de la Biología y la Educación Ambiental. IV Congreso Nacional de Investigación en Enseñanza de la Biología.

Knowledge and Didactic Knowledge of the sciences). This knowledge derives from the reflection in and on the practice (in relation to the teaching and learning of the structuring concepts of biology) that allow the student to construct explanations about the natural phenomena in a contextual way and from there, to promote the Care for self and other species, and contribute to understanding and solving socio-environmental problems from a complex perspective.

KEYWORDS: Professional Knowledge of the Teacher, Knowledge Integration, Teacher Training, Action Research, Case Study

\section{INTRODUCCIÓN}

El artículo aporta en la compresión acerca de la construcción del conocimiento profesional del profesor de biología, Fonseca (2016), plantea que en la construcción del CPPB en relación con la enseñanza de un concepto estructurante de la biología, se articulan las tres fuentes de conocimientos que se plantean en las diversas investigaciones: La fuente académica, la experiencia y el contexto. Respecto a los conocimientos que se articulan corresponden a los de la disciplina de la biología (componentes de orden sustantivo y sintáctico), los de la didáctica de las ciencias y de la biología en particular (conocimiento del currículo, de las finalidades de la enseñanza, de los procesos metodológicos, de la evaluación, del conocimiento de los estudiantes), de los conocimientos de la naturaleza de la ciencia (historia y epistemología de la ciencia) de los metadisciplinares desde la idea del constructivismo, el pensamiento complejo y la teoría critica. En relación con la fuente experiencial, se traduce en el conocimiento que el profesor construye a través de los procesos de reflexión sobre su propia práctica y de ellos deriva un saber que se legitima en su propia práctica cuando le funciona de manera razonable. $Y$ el conocimiento del contexto como la posibilidad de comprender las formas de ser estudiante en una escuela particular, la idea ser escuela de una institución singular en un municipio y región diferenciada, asunto que sin duda afecta el desarrollo de la propia práctica profesional del profesor.

Así, el CPPB se considera como producto de la integración/transformación entre conocimiento experiencial (historia de vida, contexto, experiencia) y el conocimiento académico (Conocimiento biológico y Conocimiento Didáctico de las ciencias). Este conocimiento se deriva de la reflexión en y sobre la práctica (en relación con la enseñanza y el aprendizaje de los conceptos estructurantes de la biología) que le permiten al estudiante construir explicaciones acerca de los fenómenos naturales de manera contextual y desde allí, promover el cuidado de sí mismo y de las otras especies, y aportar en la comprensión y solución de los problemas socio-ambientales desde una perspectiva compleja.

Comprender el CPPB desde la epistemología, se sitúa más bien en una reflexión epistemológica desde la práctica, es decir el conocimiento profesional del profesor deviene de la práctica misma, entendida esta como praxis, en donde se produce un conocimiento local, intersubjetivo y que se valida y legitima en tanto el propio sujeto-maestro y el propio sujetoestudiante y el contexto donde habitan se transforman, para procurar una vida más digna para todos. Así, el CPPB en relación con la enseñanza de de un concepto, se constituye en un conocimiento que le corresponde aportar en la comprensión del fenómeno de lo vivo y de la 
Bio - grafía. Escritos sobre la Biología y su Enseñanza. ISSN 2027-1034

Edición Extraordinaria. p.p. 302 - 310

Memorias del IX Encuentro Nacional de Experiencias en Enseñanza de la Biología y la Educación Ambiental. IV Congreso Nacional de Investigación en Enseñanza de la Biología.

vida y en consecuencia promover en los ciudadanos el cuidado de sí y de las otras especies. De la tesis anterior se derivan por lo menos dos asuntos sustanciales en la conceptualización del Conocimiento Profesional del Profesor de Biología (CPPB).

En este sentido, diversos investigadores han señalado el carácter integrativo del CPP, Porlán y Rivero (1998), señalan que el conocimiento práctico profesional deseable es, por tanto, el resultante de un complejo proceso de interacciones e integraciones de diferente nivel y naturaleza, organizado en torno a los problemas de la práctica profesional. "Esta integración no es una mera yuxtaposición de contenidos procedentes de diferentes fuentes, sino que implica una profunda tarea de reelaboración y transformación epistemológica y didáctica que puede realizarse en varios niveles" (Martin del Pozo, 1994 citado por Porlán y Rivero, 1998, p. 87).

Desde el programa de Tardif (2004), también se considera la idea de integración,

La relación de los docentes con los saberes no se reduce a una función de transmisión de los conocimientos. Su práctica integra estos saberes, entonces, el saber docente como un saber plural, formado por una amalgama, más o menos coherente, de saberes procedentes de la formación profesional y disciplinarios, curriculares y experienciales" (p.29).

Al igual que los anteriores autores, Valbuena (2008) plantea que el "Conocimiento Didáctico del Contenido Biológico se produce a partir del proceso de transformación, procesamiento, interrelación, e integración de diversos componentes". En este orden de ideas, Perafán (2015) señala la necesidad de comprender la categoría conocimiento profesional docente como un sistema de ideas integradas que asocia a cada uno de los saberes (saberes académicos, experienciales, rutinas y guiones, y teorías implícitas) un estatuto epistemológico fundante particular.

En este mismo sentido, Martínez y Valbuena (2013),

La práctica del profesorado constituye un ejercicio académico que es posible gracias al conocimiento que ellos poseen, dicho conocimiento a la vez se configura y reconfigura de diversos saberes y conocimientos (en tanto empíricos como formalizados) a partir de diferentes fuentes (principalmente la experiencia y la académica). Enseñar implica una práctica que trasciende la mera aplicación técnica de protocolos y el mero asistencialismo a los estudiantes, enseñar conlleva abordar la complejidad que representa la formación de sujetos; es decir, la enseñanza demanda un ejercicio profesional y por ende un conocimiento de carácter profesional. Así, los profesores constituyen sujetos de conocimiento y no simples ejecutores técnicos de los conocimientos producidos por otros (pp. 24-25)

Teniendo en cuenta lo anterior, el objetivo que se presenta en el presente texto corresponde al de: Caracterizar los conocimientos que se integran en la construcción del CPPB de un profesor en formación en relación con la enseñanza de la biodiversidad, en el espacio académico de la práctica pedagógica de la Universidad Distrital Francisco José de Caldas. 
Bio - grafía. Escritos sobre la Biología y su Enseñanza. ISSN 2027-1034

Edición Extraordinaria. p.p. 302 - 310

Memorias del IX Encuentro Nacional de Experiencias en Enseñanza de la Biología y la

Educación Ambiental. IV Congreso Nacional de Investigación en Enseñanza de la Biología.

\section{METODOLOGÍA}

El artículo se deriva del proyecto de investigación "El conocimiento profesional del profesor de biología sobre biodiversidad. un estudio de caso en la formación inicial durante la práctica pedagógica en la Universidad Distrital Francisco José de Caldas", cuya metodología se desarrolló a través de los principios de la investigación de orden interpretativa desde los principios de Vasilachis (2006), acudiendo a los planteamientos del estudio de caso Martínez (2000), Ragin (1999), Stake (1999), Yin (1994) entre otros, posibilitando la comprensión en profundidad acerca de la construcción del conocimiento profesional de profesor de biología. Por otra parte, en coherencia con el desarrollo de los objetivos de la investigación y las consideraciones acerca de la necesidad de trascender una mirada interpretativa como lo plantean, Abell (2008), se articula a la investigación los principios de la I-A, Carr (1989), Kemmis y McTaggart (1988, 2013), Kemmis et al. (2014), a través del desarrollo de los bucles de la espiral auto reflexiva.

El proceso de la investigación se desarrolló en el espacio de Práctica Pedagógica, del Proyecto Curricular Licenciatura en Biología (PCLB) de la Universidad Distrital Francisco José de Caldas, en donde el estudiante en formación (Caso Yonier) participó en la Investigación Acción, (figura 1) a través de dos bucles de la espiral auto reflexiva (planeación, acción-observación y reflexión); en el proceso se adoptó, diseñó e implementó técnicas e instrumentos para la recolección de información, unos realizados por el investigador y diligenciados por el profesor en formación (Entrevistas semi-estructuradas, ReCo, asesorías individuales y grupales, observación, filmación y transcripción de quince <15> clases) y, unos elaborados por este último (Unidad didáctica, diario de campo). Se realizó un análisis del contenido con los datos, que fueron organizados en la estructura del software cualitativo NVivo 11, que facilitó los procesos de interpretación y de construcción de conocimiento el cual se validó a través de la triangulación de técnicas e instrumentos, además de la transformación del discurso y del propio sujeto que hace parte del estudio de caso. 
Bio - grafía. Escritos sobre la Biología y su Enseñanza. ISSN 2027-1034

Edición Extraordinaria. p.p. 302 - 310

Memorias del IX Encuentro Nacional de Experiencias en Enseñanza de la Biología y la Educación Ambiental. IV Congreso Nacional de Investigación en Enseñanza de la Biología.

Figura 1. Proceso de construcción del CPPB, en relación con la enseñanza de biodiversidad a través del proceso de I-A, Bucle I-II, Conocimientos que se integran y Conocimientos que se derivan de su integración/transformación. Ver proceso en http://www.grupodeinvestigacionber.com/cppb.html

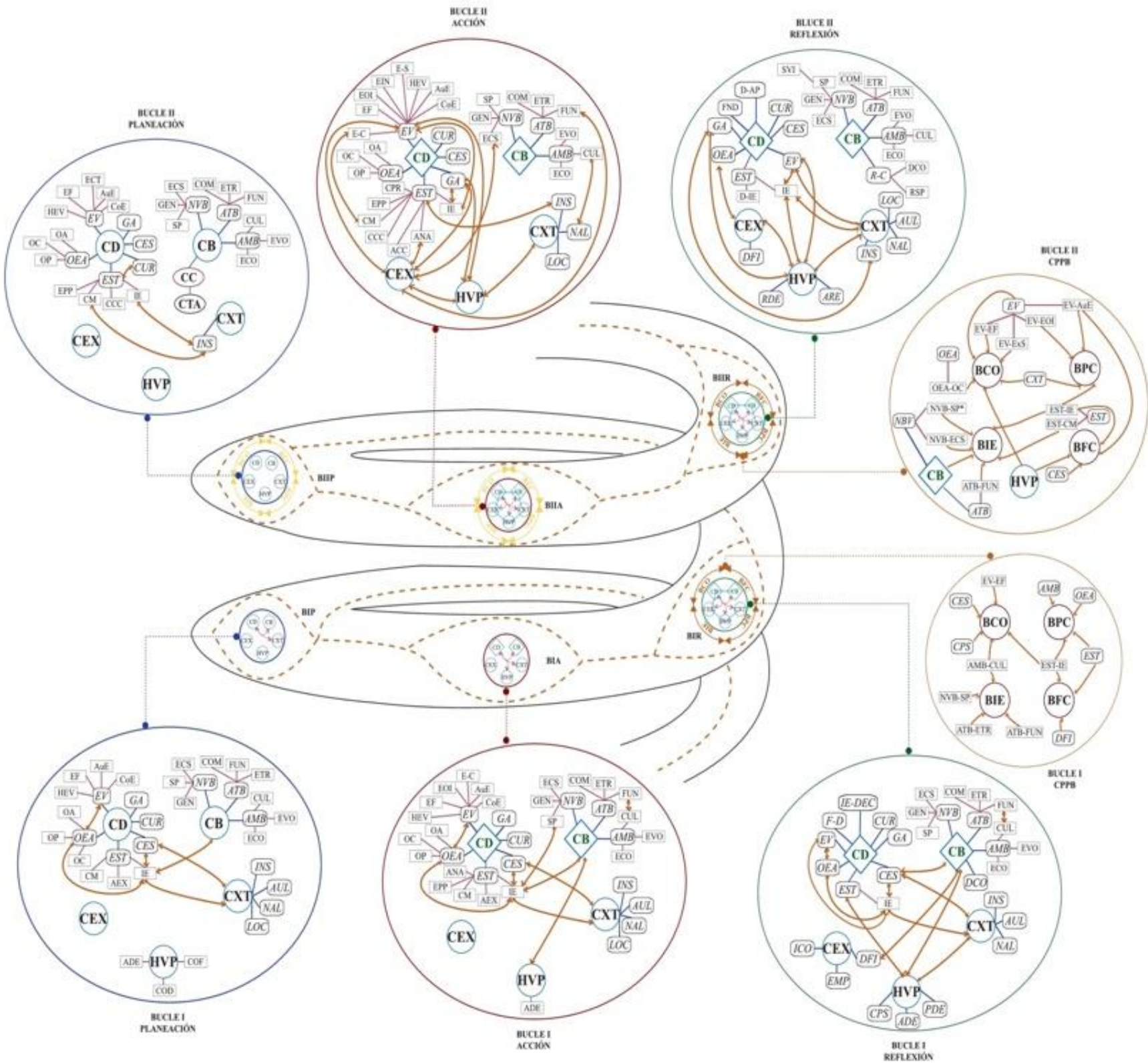

ADT: Autodeterminación; AMB: Ámbitos de Explicación; ANA: Analogías; ATB: Atributos.; AUE: Autoevaluación; AUL: Aula; CB: Conocimiento de Biodiversidad; CD: Conocimiento Didáctico; CES: Ideas de los Estudiantes; CEX: Conocimiento de la Experiencia como Interprete Ambiental; CM: Clase Magistral; CDF: Condiciones Desfavorables; COE: Coevaluación; CFV: 
Bio - grafía. Escritos sobre la Biología y su Enseñanza. ISSN 2027-1034

Edición Extraordinaria. p.p. 302 - 310

Memorias del IX Encuentro Nacional de Experiencias en Enseñanza de la Biología y la Educación Ambiental. IV Congreso Nacional de Investigación en Enseñanza de la Biología.

Condiciones Favorables; CUR: Currículo; CXT: Conocimiento del Contexto; EF: Evaluación Formativa; EOI: Evaluación como Objeto de Investigación; EPP: Explicación Práctica del Profesor; EST: Estrategias; EV: Evaluación; GA: Gestión de Aula; HEV: Heteroevaluación; HVP: Conocimiento de la Historia de Vida Propia; IE: Investigación escolar; INS: Contexto Institucional; LOC: Contexto Local; NAL: Contexto Nacional; NVB: Niveles; OA: Objetivos Actitudinales; OC: Objetivos Conceptuales; OEA: Orientaciones y Objetivos de Enseñanza; OP: Objetivos Procedimentales; RCB: Reflexión sobre el Contenido.

\section{RESULTADOS Y DISCUSIÓN}

EI CPPB en relación con la enseñanza de la biodiversidad en el caso Yonier, evidencia la importancia de la integración de cinco conocimientos: El conocimiento biológico, el Conocimiento Didáctico, el Conocimiento de su Historia de Vida, el Conocimiento de su Experiencia y el Conocimiento del contexto. Es decir, el CPPB es un conocimiento cuya naturaleza ontológica, le corresponde en sí mismo la propiedad de integración/transformación, entre conocimiento experiencial (historia de vida, contexto, experiencia como interprete ambiental) y el conocimiento académico (Conocimiento biológico y Conocimiento Didáctico de las ciencias) (Ver Figura 2). En consecuencia, de tal naturaleza se configura una epistemología particular, que se ubica en el lugar de la práctica misma.

\section{Figura 2. Cinco conocimientos que interactúan en la Práctica Profesional involucrados en el CPPB}

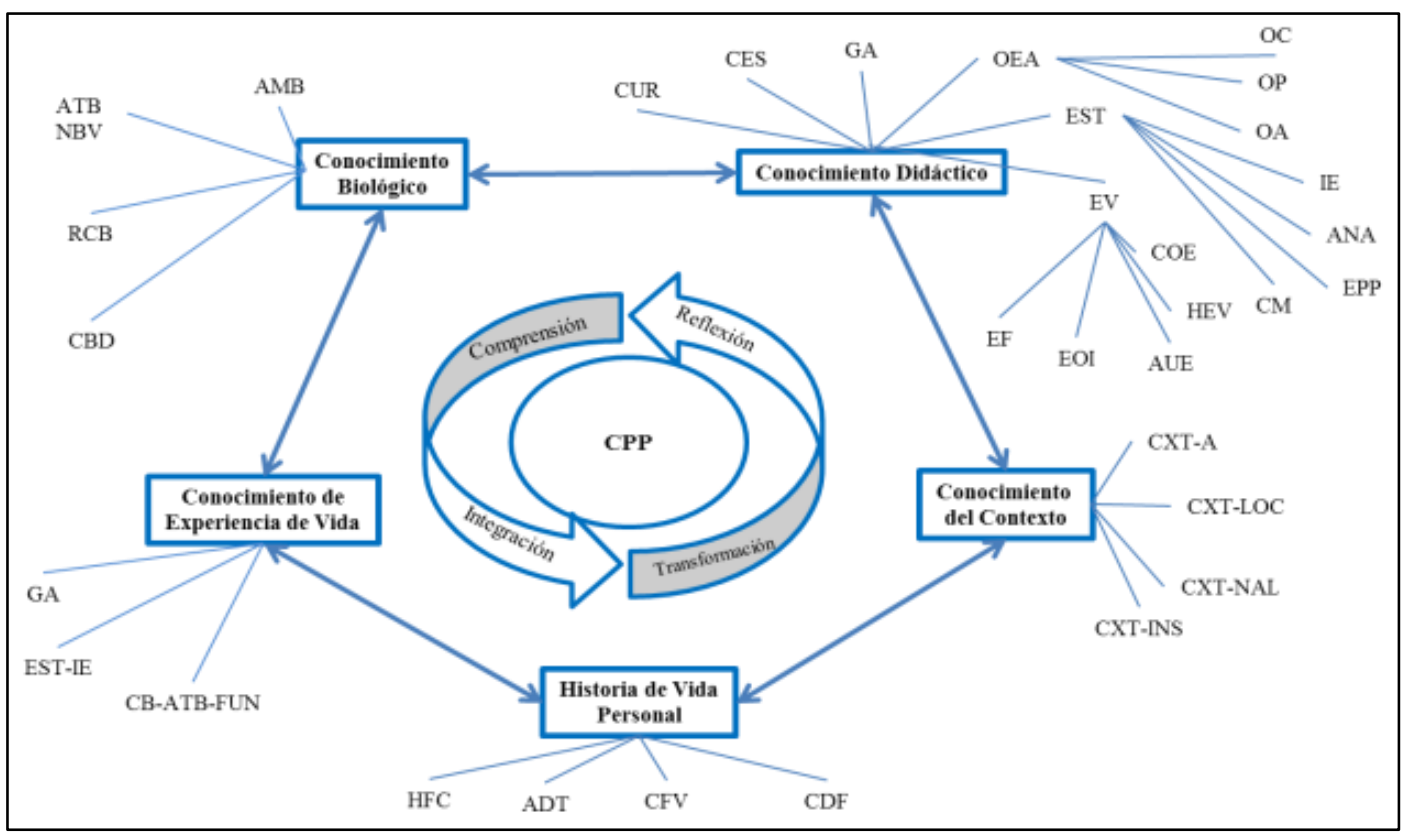

ADT: Autodeterminación; AMB: Ámbitos de Explicación; ANA: Analogías; ATB: Atributos.; AUE: Autoevaluación; AUL: Aula; CB: Conocimiento de Biodiversidad; CD: Conocimiento Didáctico; CES: Ideas de los Estudiantes; CEX: Conocimiento de la Experiencia como Interprete 
Bio - grafía. Escritos sobre la Biología y su Enseñanza. ISSN 2027-1034

Edición Extraordinaria. p.p. 302 - 310

Memorias del IX Encuentro Nacional de Experiencias en Enseñanza de la Biología y la Educación Ambiental. IV Congreso Nacional de Investigación en Enseñanza de la Biología.

Ambiental; CM: Clase Magistral; CDF: Condiciones Desfavorables; COE: Coevaluación; CFV: Condiciones Favorables; CUR: Currículo; CXT: Conocimiento del Contexto; EF: Evaluación Formativa; EOI: Evaluación como Objeto de Investigación; EPP: Explicación Práctica del Profesor; EST: Estrategias; EV: Evaluación; GA: Gestión de Aula; HEV: Heteroevaluación; HVP: Conocimiento de la Historia de Vida Propia; IE: Investigación escolar; INS: Contexto Institucional; LOC: Contexto Local; NAL: Contexto Nacional; NVB: Niveles; OA: Objetivos Actitudinales; OC: Objetivos Conceptuales; OEA: Orientaciones y Objetivos de Enseñanza; OP: Objetivos Procedimentales; RCB: Reflexión sobre el Contenido.

\section{CONCLUSIONES}

EI CPPB en relación con la enseñanza de la biodiversidad, en el caso Yonier, se evidencia la importancia de la integración de cinco conocimientos: El conocimiento biológico, el Conocimiento Didáctico, el Conocimiento de su propia historia de vida, el Conocimiento de su experiencia como interprete ambiental y el Conocimiento del contexto, en el proceso de integración se transforman en conocimientos de orden contextual y se constituyen como una propiedad emergente de las interacciones entre los conocimientos y la propia gestión de aula. Se derivan de la reflexión que se realiza sobre la práctica, en dos niveles subjetivo e intersubjetivo, con los compañeros de la práctica profesional y con el maestro titular y el asesor de práctica.

Respecto al conocimiento biológico de la biodiversidad, en el profesor en formación es posible concluir, que su desarrollo corresponde en la planeación a la organización del campo de la biodiversidad biológica, de los atributos de composición, estructura y función. Sin embargo, en la acción y reflexión se privilegiará algunos aspectos que estarán dados por el interés de Yonier para sus estudiantes; así, el Conocimiento Biológico, se transforma en un tipo de conocimiento que se relaciona con las propias finalidades de la enseñanza y del propio contexto en donde se desarrolla la práctica.

Puede concluirse, que el conocimiento didáctico en el profesor en formación se caracteriza por un alto grado de fundamentación, que se expresa en la comprensión acerca de los principios del constructivismo articulado a la investigación escolar como estrategia de enseñanza y de aprendizaje, a los procesos de evaluación como parte del proceso de aprendizaje y al reconocimiento de las ideas de los estudiantes y los procesos de complejización como fin de la enseñanza de las ciencias. En este conocimiento se integran conocimientos acerca de las finalidades de la enseñanza de la biología en clave de comprender y cuidar los seres vivos que habitan su contexto.

El conocimiento de su propia historia de vida se constituye en un elemento transversal en la configuración del CPPB en el caso Yonier, en razón de su raíz campesina lo acercaba de manera natural a los seres vivos; su vida articulada desde la autodeterminación de "salir adelante" se convierte en un aspecto que en cada clase le posibilita una idea de legitimidad, para reiterar la importancia que es posible salir adelante. La historia de vida se convierte en movilizador e integrador de los otros conocimientos, es decir su experiencia personal es conocimiento en sí mismo, la forma como ha logrado "ascender" le provee de un conocimiento personal que se traduce en una decisión ideológica-política, en considerar que sus estudiantes 
Bio - grafía. Escritos sobre la Biología y su Enseñanza. ISSN 2027-1034

Edición Extraordinaria. p.p. 302 - 310

Memorias del IX Encuentro Nacional de Experiencias en Enseñanza de la Biología y la Educación Ambiental. IV Congreso Nacional de Investigación en Enseñanza de la Biología.

tienen la oportunidad, la esperanza, y las condiciones de salir adelante. Así, este conocimiento por su naturaleza práctica y de orden existencial.

El conocimiento de su experiencia se constituye en un conocimiento vivencial en razón que debe intervenir con diferentes grupos de personas a quienes debe promover la sensibilización y cuidado del medio ambiente, esta experiencia deriva en una forma práctica de hacer, que integra en su conocimiento profesional en su práctica de aula. Es un saber de orden no institucionalizado que se construye con una intencionalidad más en el orden de la divulgación científica y de la sensibilización, en una perspectiva sistémica, características que integra en su conocimiento profesional.

El conocimiento del contexto se refiere a la comprensión de las realidades que se han construido y en tanto que es una construcción social es posible transformar, la propia manera de verse, comprenderse. Así, él comprende que las imágenes que sobre los estudiantes existen no corresponden a los propios estudiantes, solo son imágenes y no sujetos y en tal consideración es importante creer en sus estudiantes, y que la esperanza de responsabilizarse de sí mismos desde una perspectiva más propositiva contribuye a lo que él llama salir adelante.

\section{REFERENCIAS}

Abell, S. (2008). Twenty Years Later. Does Pedagogical Content Knowledge remain a useful [Veinte años más tarde. ¿El conocimiento pedagógico del contenido sigue siendo una herramienta útil?]. International Journal of Science Education, 30 (10), 1405-1416.

Carr, W. (1989). Calidad de la enseñanza e investigación acción (Edición revisada). Sevilla: Editorial Diada

Fonseca A., G. (2016) El conocimiento profesional del profesor de biología sobre biodiversidad. un estudio de caso en la formación inicial durante la práctica pedagógica en la Universidad Distrital Francisco José De Caldas. Tesis Doctoral. Universidad Distrital Francisco José de Caldas. Bogotá, Colombia.

Kemmis, S., \& MacTaggart, R. (1988). Cómo planificar la investigación-acción. Barcelona: Editorial Laertes.
Kemmis, S., \& McTaggart, R. (2013). La investigación-acción participativa: La acción comunicativa y la esfera pública. In Manual de investigación cualitativa. Vol. III, 361-439. Barcelona: Gedisa.

Kemmis, S., McTaggart, R., \& Nixon, R. (2014). The action research planner: Doing critical participatory action research. Springer Science \& Business Media.

Lera, M. J., Jensen, K., Josang, F., \& Buccoliero, E. (2007). Guía de implementación. Golden5 Programa: www. golden5.org/implementacion.

Martínez, C. A (2000) Las propuestas curriculares de los profesores sobre el conocimiento escolar: dos estudios de caso en el área de conocimiento del medio. Universidad De Sevilla.

Martínez, C. A. \& Valbuena, E. O. (2013). El conocimiento profesional de los profesores de ciencias sobre el 
Bio - grafía. Escritos sobre la Biología y su Enseñanza. ISSN 2027-1034

Edición Extraordinaria. p.p. 302 - 310

Memorias del IX Encuentro Nacional de Experiencias en Enseñanza de la Biología y la Educación Ambiental. IV Congreso Nacional de Investigación en Enseñanza de la Biología.

conocimiento escolar. Bogotá: Editorial Universidad Distrital Francisco José de Caldas.

Porlán, R., Rivero, A. \& Martín Del Pozo, R. (1998). Conocimiento profesional y epistemología de los profesores, II: Estudios empíricos y conclusiones. Enseñanza de las ciencias, 16 (2), 271278.

Ragin, Ch. \& Becker, H. (1992). What is a Case? Exploring the Foundations of Social Inquiry. Cambridge: Cambridge University Press.

Stake, R. (1999). Estudio de casos. En Denzin, N. \& Lincoln Y. (2011). Handbook of Qualitative Research. 236247, Londres: SAGE Publications.
Tardif, M. (2004). Los saberes del docente y su desarrollo profesional. Madrid; Narcea.

Valbuena, E. (2008). El Conocimiento didáctico del contenido biológico. Estudio de las concepciones disciplinares y didácticas de futuros docentes de la Universidad Pedagógica Nacional (Colombia). Madrid: Universidad Complutense

Vasillachis de Gialdino, I. (2006). Estrategias de investigación cualitativa. Barcelona, España: Editorial Gedisa S.A.

Yin, R.L. (1994), Case Study Research, Sage Publications, Thousand Oaks, CA. 